\title{
Preventive Effects of an EPA Preparation Original Article on Restenosis after Carotid Artery Stenting
}

Tomoo Ohashi, ${ }^{1}$ Yusuke Arai, ${ }^{2}$ Daichi Kato, ${ }^{2}$ Shigeru Aoyagi, ${ }^{2}$ Hirofumi Okada, ${ }^{2}$ Tomoya Yokoyama, ${ }^{1}$

Megumi Ichikawa, ${ }^{1}$ Kenta Nagai, ${ }^{1}$ Hiroaki Namatame, ${ }^{1}$ Akihiko Saida, ${ }^{1}$ Takao Hashimoto, ${ }^{2}$ and Michihiro Kono ${ }^{2}$

Objective: The purpose of this study was to investigate the preventive effects of an eicosapentaenoic acid (EPA) medication on restenosis after carotid artery stenting (CAS).

Methods: Of 134 patients (144 episodes) who underwent CAS in our hospital or affiliated institutions between November 2005 and September 2017, the subjects were 123 who could be followed-up for $\geq 30$ days after procedure by carotid ultrasonography with a mean age of 73.3 years (range: $55-90$ years). Males accounted for 106 (86.2\%) of the patients, 61 lesions $(49.6 \%)$ were symptomatic. We retrospectively compared the incidence of restenosis between the two groups: EPA-treated group $(n=43)$ and non-EPA-treated group $(n=80)$. In addition, the serum levels of fatty acid compositions had been analyzed in 97 of the 123 patients, and the relationship of the EPA/arachidonic acid (AA) ratio with perioperative ischemic complications or restenosis was examined.

Results: There was no restenosis in any of the 43 EPA-treated patients, whereas it occurred in $8(10 \%)$ of the 80 patients in the non-EPA-treated group, and the incidence of restenosis in EPA-treated group was significantly lower $(p=0.043)$ than that of non-EPA-treated group. In addition, the incidences of restenosis and perioperative ischemic complications were slightly higher in patients with a low EPA/AA ratio.

Conclusion: Although restenosis was more frequent in patients with a low EPA/AA ratio, the administration of an EPA medication may prevent restenosis in all patients who underwent CAS.

Keywords > carotid artery stenting, restenosis, eicosapentaenoic acid

\section{Introduction}

Stenosis of the cervical internal carotid artery is a risk factor for cerebral infarction associated with a stenosis-related reduction in cerebral blood flow or plaque disruption. ${ }^{1)}$ In 2008, carotid artery stenting (CAS) was approved in

\footnotetext{
${ }^{1}$ Department of Neurosurgery, Ibaraki Medical Center Tokyo Medical University, Inashiki, Ibaraki, Japan

${ }^{2}$ Department of Neurosurgery, Tokyo Medical University, Tokyo, Japan
}

Received: February 22, 2018; Accepted: May 17, 2018

Corresponding author: Tomoo Ohashi. Department of Neurosurgery, Ibaraki Medical Center Tokyo Medical University, 3-20-1 Ami-chuo, Inashiki, Ibaraki 300-0395, Japan

Email: tooohashi-nsu@umin.ac.jp

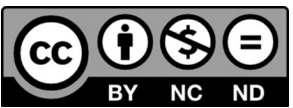

This work is licensed under a Creative Commons Attribution-NonCommercialNoDerivatives International License.

C)2018 The Japanese Society for Neuroendovascular Therapy
Japan, and it has been routinely performed. However, perioperative ischemic complications or postprocedural restenosis may occur in some cases.

It was reported that patients with a high serum eicosapentaenoic acid (EPA)/arachidonic acid (AA) ratio was significantly associated with a low incidence of major adverse cardiac events (MACE). ${ }^{2)}$ The Japan EPA Lipid Intervention Study (JELIS) indicated the preventive effects of combination therapy with an EPA preparation and statin on the onset of coronary artery events. ${ }^{3}$ Furthermore, a study reported that the administration of an EPA medication reduced intimal thickening of the rabbit carotid artery. ${ }^{4)}$ According to another study, the combination therapy of EPA and rosuvastatin stabilized vulnerable carotid plaques. ${ }^{5)}$ On the other hand, a study suggested the potential of vulnerable carotid plaques as a risk factor for restenosis. $\left.{ }^{6}\right)$ In this study, we investigated whether the administration of an EPA medication to patients with a low EPA/AA ratio or vulnerable plaques prevents restenosis after CAS. 
Table 1 Patient profile and condition

\begin{tabular}{lc} 
Number of lesions (n) & 123 \\
Age (years) & $73.3[55-90]$ \\
Gender (male/female) & $106 / 17$ \\
Symptomatic lesions (n, \%) & $61(49.6)$ \\
Stenosis rate (\%) & $74.8[37-99]$ \\
Vulnerable plaques (n, \%) & $23(18.7)$ \\
\hline
\end{tabular}

\section{Methods}

Of 134 patients (144 episodes) who underwent CAS in our hospital or affiliated institutions between November 2005 and September 2017, the subjects were 123 who could be followed-up for $\geq 30$ days after surgery by carotid ultrasonography, with a mean age of 73.3 years (range: 55-90 years). Males accounted for 106 (86.2\%) of the patients, 61 lesions $(49.6 \%)$ were symptomatic. We retrospectively compared the incidence of restenosis between the two groups: EPA-treated group $(n=43)$ and non-EPAtreated group $(n=80)$ (Table 1).

In 97 of the 123 patients, the serum levels of fatty acid compositions had been analyzed in fasting blood samples on the morning before starting EPA medication. According to subanalysis in the JELIS, these patients were divided into three groups based on the EPA/AA ratio: $\geq 1.06$ (H group), 0.56-1.05 (M group), and $\leq 0.55$ (L group) (Table 2).

The plaque characteristics of carotid artery stenotic lesions were examined, and plaques that met any of the following were defined as vulnerable plaques: 1) hypoechoic or mobile plaque on carotid ultrasonography; 2) those with a plaque/sternocleidomastoid signal intensity ratio of $\geq 1.25$ on T1-weighted black-blood (BB) $\mathrm{MRI}^{7)}$; and 3 ) those showing white plaque sign on time-of-flight magnetic resonance angiography (MRA-TOF). ${ }^{8}$

Postprocedural restenosis was defined as cases of area stenosis $\geq 50 \%$ and peak systolic velocity (PSV) $\geq 200 \mathrm{~cm} /$ sec by carotid ultrasonography.

CAS was performed for all patients by the same team using the standardized method. For patients in the early period (August 2003 to January 2008), primarily closedcell stent was used with distal balloon protection technique in the intermediate period (February 2008 to March 2011), primarily open-cell stent was used with distal filter protection technique, and in the late period (after April 2011), tailored CAS, in which a device is selected based on the evaluation of plaque characteristics, was introduced.

Because serum fatty acid composition measurement was approved in March 2011, of the late-period patients, EPA medications (ethyl eicosapentaenoate, $900 \mathrm{mg} \times 2$,
Table 2 Number of patients with respect to the EPA/AA ratio (number of patients with restenosis) and incidence of EPA medication $(n=97)$

\begin{tabular}{ccccc} 
& \multicolumn{5}{c}{ EPA/AA } & \\
& H group & M group & L group & Unknown \\
& $(\geq 1.06)$ & $(0.56-1.05)$ & $(\leq 0.55)$ & $(n=26)$ \\
& $(n=8)$ & $(n=23)$ & $(n=66)$ & \\
EPA medication & & & & \\
Yes $(n=43)$ & $0[0]$ & $9[0]$ & $28[0]$ & $6[0]$ \\
No $(n=80)$ & $8[0]$ & $14[1]$ & $38[7]$ & $20[0]$ \\
\hline
\end{tabular}

AA: arachidonic acid; EPA: eicosapentaenoic acid

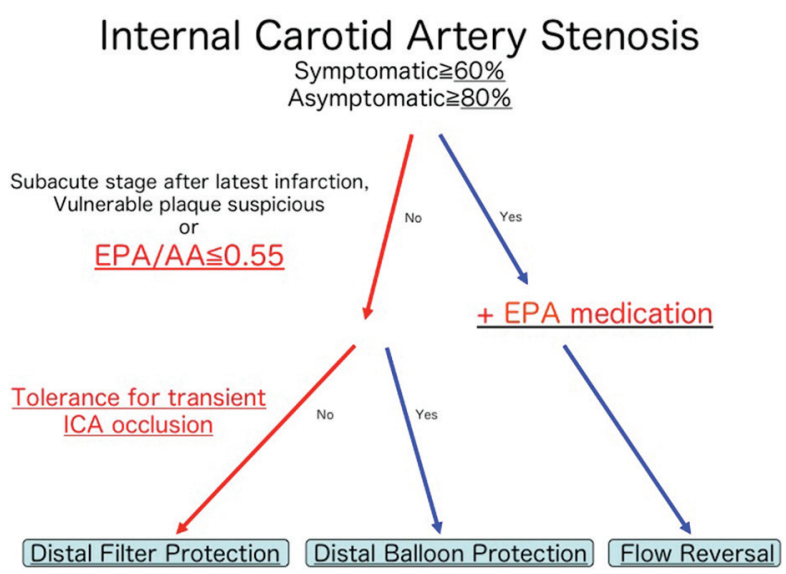

Fig. 1 CAS algorithm of our hospital after April 2011. AA: arachidonic acid; CAS: carotid artery stenting; EPA: eicosapentaenoic acid; ICA: internal carotid artery

immediately after breakfast and dinner, or omega-3 fatty acid ethyl, $2 \mathrm{~g} \times 1$, immediately after breakfast) were orally administered to those with subacute cerebral infarction, those in whom vulnerable plaques were suspected before procedure, and those assigned to the L group (EPA/AA ratio), early during the periprocedural period (Fig. 1).

For statistical analysis, we used IBM SPSS Statistics (IBM Corporation, Armonk, NY, USA). A p value of 0.05 (paired) was regarded as significant. The protocol of this clinical study was approved by the Ethics Review Board of Tokyo Medical University Ibaraki Medical Center.

\section{Results}

Restenosis was observed in $8(6.5 \%)$ of the 123 patients. There were no significant differences in background factors, such as the stenotic rate, age, sex, mode of onset, or rate of statin medication, between the restenosis and non-restenosis groups (Table 3).

Concerning EPA drug administration, there was no restenosis in any of 43 EPA-treated patients, whereas it occurred in $8(10 \%)$ of 80 non-EPA-treated patients. When evaluating restenotic events concerning EPA drug administration 
Table 3 Baseline clinical characteristics

\begin{tabular}{|c|c|c|c|}
\hline & \multicolumn{2}{|c|}{ Restenosis } & \multirow{2}{*}{$p$ value } \\
\hline & Yes $(n=8)$ & No $(n=115)$ & \\
\hline Stenotic rate (\%) & $79.0[70-88]$ & 74.5 [37-99] & 0.4 \\
\hline Gender (male/female) & $8 / 0$ & $98 / 17$ & 0.24 \\
\hline Symptomatic lesions (n, \%) & $5(62.5)$ & $56(48.7)$ & 0.45 \\
\hline Age (years) & 71.9 [60-83] & $73.4[55-90]$ & 0.55 \\
\hline Statin (n, \%) & $5(62.5)$ & $67(58.3)$ & 0.81 \\
\hline Cilostazol (n, \%) & $1(12.5)$ & $27(23.5)$ & 0.47 \\
\hline Pioglitazone (n, \%) & $0(0.0)$ & $2(1.7)$ & 0.71 \\
\hline DM (n, \%) & $4(50.0)$ & $34(29.6)$ & 0.23 \\
\hline $\mathrm{HT}(\mathrm{n}, \%)$ & $6(75.0)$ & 63 (54.8) & 0.27 \\
\hline $\mathrm{HL}(\mathrm{n}, \%)$ & $3(37.5)$ & 53 (46.1) & 0.64 \\
\hline Closed-cell stent (n, \%) & $5(62.5)$ & $74(64.3)$ & 0.92 \\
\hline EPA/AA, mean (range) $(※ n=97)$ & $0.41[0.21-0.85]$ & $0.55[0.05-1.8]$ & 0.31 \\
\hline
\end{tabular}

AA: arachidonic acid; DM: diabetes; EPA: eicosapentaenoic acid; HL: hyperlipidemia; HT: hypertension

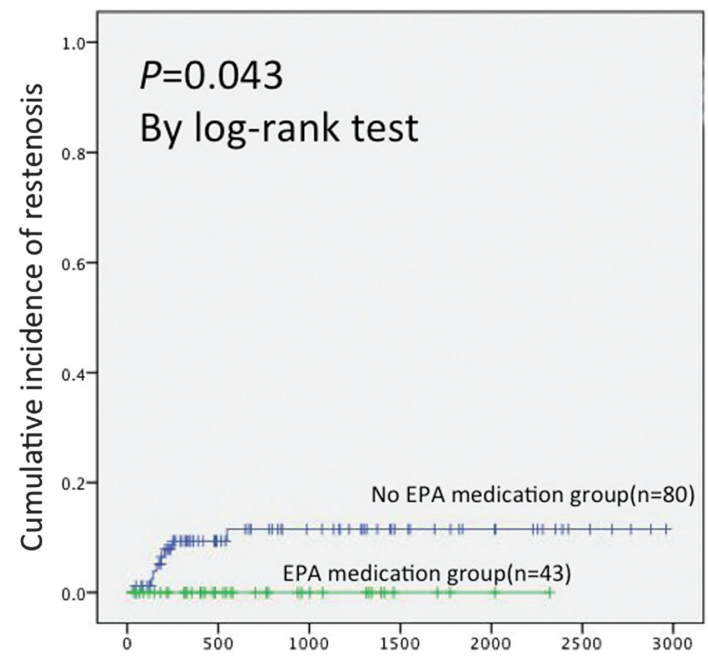

(Days)

Fig. 2 Kaplan-Meier survival curves: EPA vs. no EPA about restenosis after CAS. CAS: carotid artery stenting; EPA: eicosapentaenoic acid

using the Kaplan-Meier method, the administration of EPA preparations significantly reduced the incidence of restenosis $(p=0.043)$ (Fig. 2). In 22 patients in whom the serum levels of fatty acid compositions could be measured after the start of administration, the mean EPA/AA ratio increased by $0.51(\mathrm{t}=3.37, \mathrm{p}=0.001)$.

Of 97 patients in whom the serum levels of fatty acid compositions, the EPA/AA ratio of symptomatic cases was significantly lower than that of asymptomatic cases $(0.41 \pm$ 0.23 vs. $0.64 \pm 0.43, p<0.005)$. In a group with a low EPA/ AA ratio (L group), restenosis was noted in 7 (10.6\%) of 66 patients (Table 2). However, these were included in 38 non-EPA-treated patients, and there was no restenosis in any of 28 EPA-treated patients $(p=0.027)($ Fig. 3).

The rate of patients in whom vulnerable plaques were clarified before procedure or those with postprocedural

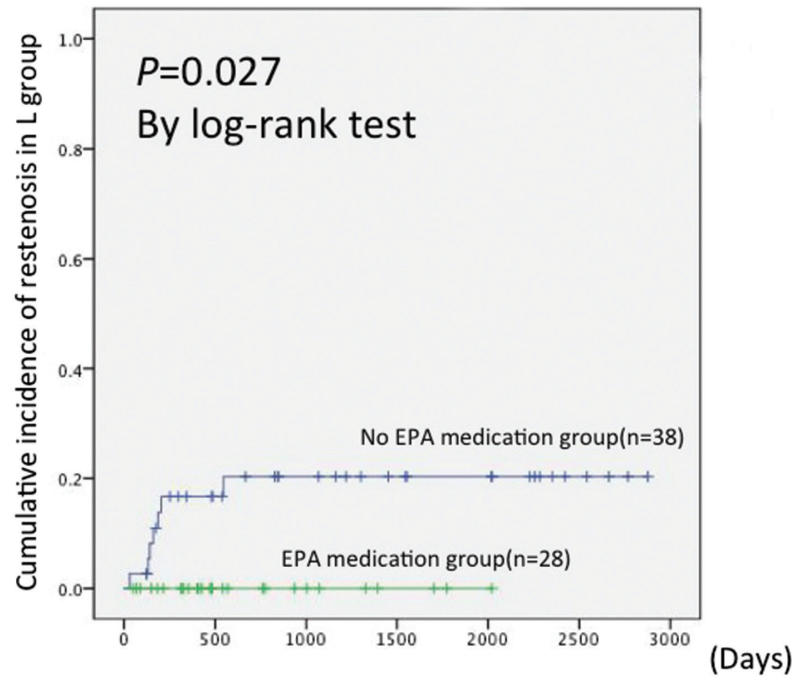

Fig. 3 Kaplan-Meier survival curves: EPA vs. no EPA in L group about restenosis after CAS. CAS: carotid artery stenting; EPA: eicosapentaenoic acid

restenosis was significantly higher in the L group. The rate of patients with positive findings on postprocedural diffusion-weighted imaging (DWI) was slightly higher in L group. In addition, minor stroke occurred only in the L group (Table 4).

Concerning the clinical background of the EPA-treated and non-EPA-treated groups, there were significant differences in the stenotic rate, rate of cilostazol medication, incidence of hyperlipidemia, and EPA/AA ratio. The rate of patients for whom a closed-cell stent was selected was slightly higher in the EPA-treated group (Table 5).

\section{Discussion}

A study reported that there was a correlation between intimal carotid thickening and the risk of stroke. ${ }^{9)}$ However, several 
Table 4 Comparison of ischemic complications between the $L$ group and other groups

\begin{tabular}{lccc} 
& L group $(n=66)$ & Other groups $(n=57)$ & $p$ \\
Restenosis $(n, \%)$ & $7(10.6)$ & $1(1.8)$ & 0.0471 \\
Vulnerable plaque $(n, \%)$ & $17(25.8)$ & $6(12.9)$ & 0.0307 \\
DWI positive $(n, \%)$ & $13(19.7)$ & $5(6.5)$ & 0.0874 \\
Minor stroke (n, \%) & $2(3.0)$ & 0 & 0.1851 \\
\hline
\end{tabular}

DWI: diffusion-weighted imaging

Table 5 Clinical background of the EPA-treated and non-EPA-treated groups

\begin{tabular}{lccc} 
& \multicolumn{2}{c}{ EPA medication } & p value \\
& Yes $(\mathrm{n}=43)$ & No $(\mathrm{n}=80)$ & \\
Stenotic rate $(\%)$ & $69.9[43-99]$ & $77.5[37-99]$ & 0.0056 \\
Gender (male/female) & $35 / 8$ & $71 / 9$ & 0.26 \\
Symptomatic lesions $(\mathrm{n}, \%)$ & $21(48.8)$ & $40(50.0)$ & 0.9 \\
Age (years) & $69.9[55-89]$ & $73.9[57-90]$ & 0.16 \\
Statin (n, \%) & $28(65.1)$ & $44(55.0)$ & 0.28 \\
Cilostazol $(\mathrm{n}, \%)$ & $15(34.9)$ & $13(16.3)$ & 0.0188 \\
Pioglitazone (n, \%) & $1(2.3)$ & $1(1.3)$ & 0.65 \\
DM (n, \%) & $16(37.2)$ & $22(27.5)$ & 0.27 \\
HT (n, \%) & $23(53.5)$ & $46(57.5)$ & 0.67 \\
HL (n, \%) & $26(60.5)$ & $30(37.5)$ & 0.0147 \\
Closed-cell stent $(\mathrm{n}, \%)$ & $32(74.4)$ & $47(58.8)$ & 0.0839 \\
EPAAA, mean (range) $(* \mathrm{n}=97)$ & $0.43[0.05-0.92]$ & $0.6[0.14-1.8]$ & 0.023 \\
\hline
\end{tabular}

AA: arachidonic acid; DM: diabetes; EPA: eicosapentaenoic acid; HT: hyperlipidemia; HL: hypertension

studies indicated that cilostazol, statins, pioglitazone, and EPA medications inhibited intimal carotid thickening. ${ }^{4,10-12)}$ In particular, these drugs may reduce or stabilize vulnerable plaques involving intra-plaque thrombi or necrotic tissue..$^{5)}$ The incidence of periprocedural ischemic complications after CAS is high in patients with vulnerable plaques, and these drugs may reduce such complications.

On the other hand, the incidence of restenosis after CAS is reportedly $2 \%-7 \% .{ }^{13)}$ The outgrowth of smooth muscle cells and extracellular matrix deposition may lead to neointimal hyperplasia. In addition, soft fibro-fatty lesions may progress to in-stent stenosis. $\left.{ }^{6}\right)$ Takigawa et al. ${ }^{14)}$ reported the preventive effects of cilostazol administration on restenosis. The plaque-stabilizing or -reducing effects of the above drugs may contribute to the prevention of restenosis.

EPA contained in fish oil or health foods is classified as a $\omega 3$ polyunsaturated fatty acid (PUFA), as reported for docosahexaenoic acid (DHA). It acts on Resolvin E1/E2, thromboxane $\mathrm{A} 3$, prostaglandin E3/D3/I3, and leukotriene $\mathrm{B} 5 / \mathrm{C} 5 / \mathrm{D} 5 / \mathrm{E} 5$, exhibiting anti-inflammatory actions. Although its action mechanism remains to be clarified, arterial elasticity-maintaining, serum lipid-reducing, and platelet aggregation-inhibiting actions may be involved. ${ }^{15)}$ Sawada et al. ${ }^{5)}$ reported that EPA/rosuvastatin administration led to significant plaque stabilization and plaque regression on intravascular ultrasound (IVUS) in the vulnerable plaque group with a carotid plaque/sternocleidomastoid ratio of $\geq 2$ on MRI using the two-dimensional (2D) spin-echo BB method. In our series, neither perioperative ischemic complications nor restenosis was observed after changing the therapeutic strategy. In addition to the introduction of tailored CAS, qualitative and quantitative changes in plaques related to the administration of EPA medications may have contributed to favorable results. In particular, before changing the therapeutic strategy, we noticed that the incidence of ischemic complications in the L group was slightly higher, resulting in a change of the device strategy and intensified medical treatment to overcome it. After changing the therapeutic strategy, EPA preparations were administered primarily to the $\mathrm{L}$ group, but, overall, the results for the $\mathrm{L}$ group were unfavorable; therefore, if the therapeutic strategy had not been changed, the results might have been poorer.

Concerning the clinical background of the EPA-treated and non-EPA-treated groups, the percent stenosis was significantly higher in the non-EPA-treated group, but there was no difference in the percent stenosis between the restenosis and non-restenosis groups, as shown in Table 3. We do not consider that a high stenotic rate before procedure was disadvantageous for the non-EPA-treated group with respect to the development of post-treatment restenosis. A larger number of patients belonging to the $\mathrm{L}$ group were included in the EPA-treated group although there was no significant difference ( 65.1 vs. $47.5 \%$, respectively, $\mathrm{p}=$ 0.0617). Considering that a similar rate of patients with symptomatic lesions were included in the EPA-treated group despite a low stenotic rate, lesions may become 
symptomatic if the EPA/AA ratio is low despite a low stenotic rate. Furthermore, in the EPA-treated group, cilostazol and a closed-cell stent were selected for a high rate of patients. However, the latter may have been associated with the timing of treatment and social background. The combination of an EPA, cilostazol, and a closed-cell stent may be more effective in preventing restenosis although there was no difference between the restenosis and nonrestenosis groups, as shown in Table 3.

The serial changes in the EPA/AA ratio support the administration of EPA medications primarily to the L group, but the mean EPA/AA ratio increased by 0.51 in 22 patients in whom the serum levels of fatty acid compositions could be measured after the start of administration $(\mathrm{t}=3.37, \mathrm{p}=$ 0.001). Since April 2011, when the administration of EPA preparations and tailored CAS were started, there has been no ischemic complication or restenosis. For 7 of 8 patients with restenosis, additional stenting and additional therapy with EPA preparations were performed, and there has been no recurrence in any patient. The administration of EPA preparations increased the EPA/AA ratio, which may have contributed to the results of this study.

\section{Conclusion}

The administration of EPA medications decreased the incidence of restenosis after CAS. In addition to preprocedural plaque evaluation, serum fatty acid composition analysis and EPA drug administration may be important for the prevention of periprocedural ischemic complications and postprocedural restenosis.

\section{Acknowledgments}

We especially thank Prof. Watanabe (Director of the General Health Medical Center, Yokohama University of Pharmacy) and Prof. Honda (Director of the Cooperative Research Center, Tokyo Medical University Ibaraki Medical Center) for their advice.

\section{Disclosure Statement}

There is no conflict of interest for the first author and coauthors.

\section{References}

1) Kitamura $\mathrm{A}$, Iso $\mathrm{H}$, Imano $\mathrm{H}$, et al: Carotid intima-media thickness and plaque characteristics as a risk factor for stroke in Japanese elderly men. Stroke 2004; 35: 2788-2794.
2) Domei T, Yokoi H, Kuramitsu S, et al: Ratio of serum n-3 to n-6 polyunsaturated fatty acids and the incidence of major adverse cardiac events in patients undergoing percutaneous coronary intervention. Circ J 2012; 76: 423-429.

3) Saito Y, Yokoyama M, Origasa H, et al: Effects of EPA on coronary artery disease in hypercholesterolemic patients with multiple risk factors: sub-analysis of primary prevention cases from the Japan EPA lipid intervention study (JELIS). Atherosclerosis 2008; 200: 135-140.

4) Iwamoto T, Ogawa K, Sasaki A, et al: [Effect of ethyl icosapentaenoate (EPA) on intimal thickening in cuff-sheathed model of a rabbit carotid artery]. $J$ Atheroscler Thromb 1993; 21: 443-448. (in Japanese)

5) Sawada M, Tanabe J, Yasogawa Y, et al: [EPA and rosuvastatin stabilize the unstable carotid plaques: a prospective randomized clinical trial]. Surg Cerebral Stroke 2013; 41: 39-45. (in Japanese)

6) Tamakawa N, Sakai H, Nishimura Y: [Prediction of restenosis progression after carotid artery stenting using virtual histology IVUS]. JNET 2008: 2: 193-200. (in Japanese)

7) Koyanagi M, Yoshida K, Kishida N, et al: [Carotid artery stenting based on plaque characteristics using magnetic resonance plaque imaging]. JNET 2012; 6: 240-244. (in Japanese)

8) Yoshimura S, Yamada K, Kawasaki M, et al: High-intensity signal on time-of-flight magnetic resonance angiography indicates carotid plaques at high risk for cerebral embolism during stenting. Stroke 2011; 42: 3132-3137.

9) Bots ML, Hoes AW, Koudstaal PJ, et al: Common carotid intima-media thickness and risk of stroke and myocardial infarction: the Rotterdam Study. Circulation 1997; 96: 1432-1437.

10) Shinoda-Tagawa $T$, Yamasaki $Y$, Yoshida $S$, et al: A phosphodiesterase inhibitor, cilostazol, prevents the onset of silent brain infarction in Japanese subjects with Type II diabetes. Diabetologia 2002; 45: 188-194.

11) Crouse JR, Raichlen JS, Riley WA, et al: Effect of rosuvastatin on progression of carotid intima-media thickness in low-risk individuals with subclinical atherosclerosis: the METEOR Trial. JAMA 2007; 297: 1344-1353.

12) Mazzone T, Meyer PM, Feinstein SB, et al: Effect of pioglitazone compared with glimepiride on carotid intimamedia thickness in type 2 diabetes: a randomized trial. JAMA 2006; 296: 2572-2581.

13) Wholey MH, Wholey M, Mathias K, et al: Global experience in cervical carotid artery stent placement. Catheter Cardiovasc Interv 2000; 50: 160-167.

14) Takigawa $T$, Matsumaru $Y$, Hayakawa M, et al: Cilostazol reduces restenosis after carotid artery stenting. $J$ Vasc Surg 2010; 51: 51-56.

15) Dona M, Fredman G, Schwab JM, et al: Resolvin E1, an EPA-derived mediator in whole blood, selectively counterregulates leukocytes and platelets. Blood 2008; 112: 848-855. 